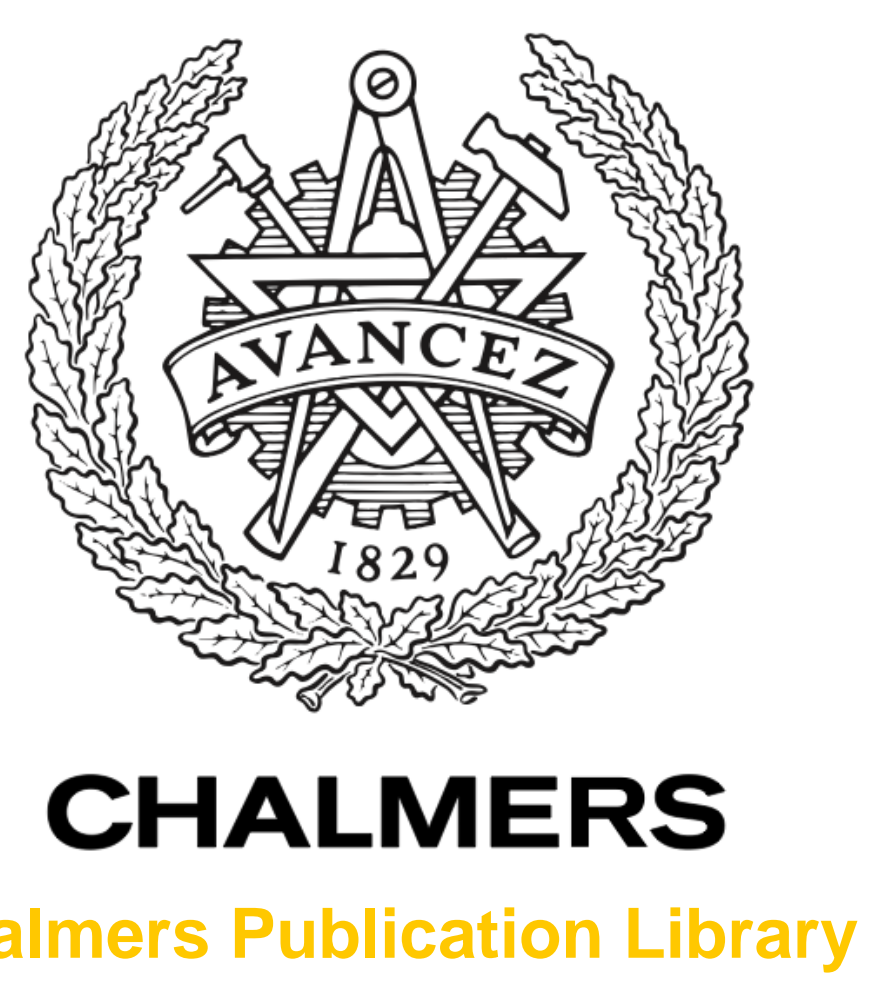

Chalmers Publication Library

\title{
The single-scattering properties of black carbon aggregates determined from the geometric-optics surface-wave approach and the T-matrix method
}

This document has been downloaded from Chalmers Publication Library $(\mathrm{CPL})$. It is the author's version of a work that was accepted for publication in:

Journal of Quantitative Spectroscopy \& Radiative Transfer (ISSN: 0022-4073)

Citation for the published paper:

Takano, Y. ; Liou, K. ; Kahnert, M. (2013) "The single-scattering properties of black carbon aggregates determined from the geometric-optics surface-wave approach and the T-matrix method". Journal of Quantitative Spectroscopy \& Radiative Transfer, vol. 125 pp. 51-56.

http://dx.doi.org/10.1016/j.jqsrt.2013.04.006

Downloaded from: http://publications.lib.chalmers.se/publication/181478

Notice: Changes introduced as a result of publishing processes such as copy-editing and formatting may not be reflected in this document. For a definitive version of this work, please refer to the published source. Please note that access to the published version might require a subscription. 


\title{
The single-scattering properties of black carbon aggregates determined from the geometric-optics surface-wave approach and the T-matrix method
}

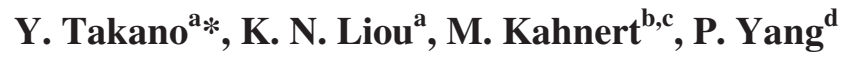 \\ ${ }^{a}$ Joint Institute for Earth System Science and Engineering, and Department of Atmospheric and \\ Oceanic Sciences, University of California, Los Angeles, CA 90095, USA \\ ${ }^{\mathrm{b}}$ Swedish Meteorological and Hydrological Institute, 60176 Norrköping, Sweden \\ ${ }^{\mathrm{c} D e p a r t m e n t}$ of Earth and Space Science, Chalmers University of Technology, 41296 \\ Gothenburg, Sweden \\ ${ }^{\mathrm{d}}$ Department of Atmospheric Sciences, Texas A\&M University, College Station, TX 77845, USA
}

\begin{abstract}
The single-scattering properties of eight black carbon (BC, soot) fractal aggregates, composed of primary spheres from 7 to 600, computed by the geometric-optics surface-wave (GOS) approach coupled with the Rayleigh-Gans-Debye (RGD) adjustment for size parameters smaller than approximately 2 , are compared with those determined from the superposition T-matrix method. We show that under the condition of random orientation, the results from GOS/RGD are in general agreement with those from T-matrix in terms of the extinction and absorption cross sections, the single-scattering co-albedo, and the asymmetry factor. When compared with the specific absorption $\left(\mathrm{m}^{2} / \mathrm{g}\right)$ measured in the laboratory, we illustrate that using the observed radii of primary spheres ranging from 3.3 to $25 \mathrm{~nm}$, the theoretical values determined from GOS/RGD for primary sphere numbers of 100-600 are within the range of measured values. The GOS approach can be effectively applied to aggregates composed of a large number of primary spheres (e.g., > 6000) and large size parameters (>>2) in terms of computational efforts.
\end{abstract}

\section{Keywords:}

Black carbon

Soot

Aggregated aerosol

Geometric optics and surface wave

Rayleigh-Gans-Debye scattering

T-matrix method

\footnotetext{
*Corresponding author. Tel.: +1 310794 9832; fax: +1 3107949796.

Email address: ytakano@atmos.ucla.edu (Y. Takano).
} 


\section{Introduction}

Aerosols are an important factor in regulating climate and weather processes. Black carbon (BC or soot) emitted from anthropogenic combustion sources has a potential effect on regional and global climate change by means of absorbing incoming sunlight [1-3]. However, determination of the absorption and scattering properties of soot is extremely difficult due to its agglomerated structure and inhomogeneous composition.

We developed a geometric optics approach coupled with surface-wave contributions for light scattering by homogeneous spheres and spheres with a shell-core structure [4]. The geometric-optics surface-wave theory, referred to as GOS, for light scattering by spheres considers the surface-wave contribution as a perturbation term to the geometric-optics core that includes the Fresnel reflection-refraction and Fraunhofer diffraction. The hit-and-miss Monte Carlo photon tracing program for homogeneous particles was extended to concentrically stratified spheres [5]. Results computed from GOS and the "exact" Lorenz-Mie type solution for spheres and rigorous numerical approaches for ice plates and columns revealed close comparisons in terms of the extinction cross section, single-scattering albedo, and asymmetry factor. Liou et al. [6] further developed computer-generated aggregates by stochastic processes using homogeneous and shell-core spheres with and without rough surfaces as the building blocks and conducted the computation of the single-scattering properties of aggregated soot particles with closed- and open-cell structures using the GOS approach. Application has been made to the study of radiative forcings produced by contrail cirrus contaminated by soot particles internally and externally mixed with small ice crystals [7].

The T-matrix method, which was originally implemented for homogeneous star-shaped particles with axisymmetric [8,9] and non-axisymmetric [10,11] geometries, has been extended to aggregated particles by means of a superposition approach [12]. The superposition T-matrix method solves Maxwell's equations for fractal aggregates, i.e., a cluster of spheres. Optical properties can be computed for particles in either fixed or random orientations. For the latter case, an analytic orientation-averaging procedure has been followed. Numerical problems and high CPU-time demands can become an issue for particles much larger than the incident wavelength and for large numbers of primary particles in the aggregate. For the latest parallelized implementation of the code [13], stable results have been reported for particles consisting of up to 3000 primary spheres. 
In this note, we present comparison of the single-scattering properties of soot aggregates computed from GOS and the results determined from the superposition T-matrix method [12] presented in various studies (e.g. [14-16]). The accuracy of the T-matrix method applied to BC aggregates has been extensively tested by comparisons to discrete dipole computations [17] and by use of the reciprocity condition [17-19]. In the course of this comparison, we developed a Rayleigh-Gans-Debye (RGD) approximation for aggregates with volume-equivalent size parameters less than approximately 2 to supplement limitations of the GOS approach. In Section 2 , the computed single-scattering properties derived from GOS/RGD and T-matrix are shown and discussed, followed by the concluding remarks denoted in Section 3. The RGD approximation is presented in the Appendix.

\section{Computational results and discussions}

Black carbon (or soot) aggregates produced by combustion usually have fractal structures. In this work, we have adopted the diffusion limited cluster aggregates (DLCA, [20]) composed of $N_{s}$ homogeneous primary spheres of equal radius $a$. Using random numbers, DLCA can be generated stochastically in a different way as compared to the diffusion limited aggregates (DLA) presented in Liou et al. [6]. It allows collisions among agglomerates in addition to collisions between primary spheres undergoing a random walk and agglomerates. For this reason, its fractal dimension of 1.82 is smaller than that of DLA, which has a value of 2.27. Following Kahnert $[15,16]$, we have assumed eight different fractal aggregates composing of primary spheres $N_{s}$ of 7,23,54,105, 180,284, 423, and 600. The corresponding volume-equivalent radii $r_{v}$ are given by $a\left(N_{s}\right)^{1 / 3}$ with $a=25 \mathrm{~nm}$, unless stated otherwise. Figure 1 depicts three examples of the fractal aggregates composing of $N_{s}$ of 54,284, and 600.

In the course of comparison between GOS and the superposition T-matrix method, we find that the results computed from GOS diverge from those obtained from T-matrix for size parameters smaller than $\sim 2$. For this reason, we have developed a modification of the RayleighGans scattering approximation $[21,22]$ for a sphere which incorporates Debye's form factor theory [23] for aggregates, referred to as the RGD adjustment presented in the Appendix. The RGD approximation is generally valid for size parameters smaller than $\sim 2$ and it compensates for limitations of the GOS approach. The unification of the two is shown to be a very useful method for the calculation of the single-scattering properties of soot aggregates. 
Figure 2 illustrates comparison of the extinction and absorption cross sections $C_{\text {ext }}$ and $C_{\mathrm{abs}}$, the asymmetry factor $g$, and the single-scattering co-albedo $(1-\varpi)$ computed from GOS/RGD and the T-matrix method. In the calculations, the complex index of refraction for soot at a visible wavelength $\lambda$ of $533.2 \mathrm{~nm}$ is taken to be $1.76-i 0.63$. The size parameter for the volume-equivalent sphere $x_{v}\left(=2 \pi r_{v} / \lambda\right)$ corresponding to $N_{s}$ is displayed at the top of each panel. The results cover a range of $N_{s}$ from 7 to $600[15,16]$. The extinction cross section increases with the number of spheres $N_{s}$ almost linearly. Differences between the two are less than $10 \%$. Results of the single-scattering co-albedo, the ratio of the absorption cross section to the extinction cross section, obtained from the two methods show differences of less than $3 \%$. The asymmetry factor results determined from the two methods exhibit close comparison.

In Fig. 3, we illustrates comparison of the scattering phase matrix for randomly oriented soot aggregates of $N_{s}=105$, corresponding to a size parameter of 1.39 , between RGD and Tmatrix. The phase function $P_{11}$ depicts a peak around scattering angle of $0^{\circ}$ resulting from diffraction and becomes flat at backscattering directions. The degree of linear polarization $P_{12} / P_{11}$ has a maximum value 1.0 at the scattering angle of $90^{\circ}$, which is the same as the Rayleigh scattering results. The values of $P_{22} / P_{11}$ are close 1 , while $P_{43} / P_{11}$ is $\sim 0$. The element $P_{33} / P_{11}$ is essentially equal to $P_{44} / P_{11}$ at all scattering angles. Results computed for all matrix elements from the two approaches reveal close comparison.

Figure 4 illustrates comparison of the specific absorption $\alpha_{a}\left(\mathrm{~m}^{2} / \mathrm{g}\right)$ between theoretical calculations and laboratory measured values presented by Sheridan et al. [24] at $\lambda=530 \mathrm{~nm}$ and compiled by Bond and Bergstrom [25] at $\lambda=550 \mathrm{~nm}$. The measured $\alpha_{a}$ has a value of $7.5 \pm 1.2$ $\mathrm{m}^{2} / \mathrm{g}$. It is defined by $C_{\text {abs }} / \rho V$, where $\rho$ is the soot density taken to be $1.8 \mathrm{~g} / \mathrm{cm}^{3}$ [25] and $V$ is aggregate volume. According to Wentzel at el. [26] and Bond and Bergstrom [25], the radii of primary spheres range from 3.3 to $25 \mathrm{~nm}$. However, the total number of primary spheres was not estimated from the aggregate photo. Using the preceding two radii, we obtained $\alpha_{a}(a=3.3 \mathrm{~nm})$ of $\sim 9.9 \mathrm{~m}^{2} / \mathrm{g}$ and $\alpha_{a}(a=25 \mathrm{~nm})$ of $\sim 5.8 \mathrm{~m}^{2} / \mathrm{g}$ from GOS/RGD (two red lines), which are comparable with the range of the measured values. The specific absorption appears to be largely independent of the number of primary spheres between 100 and 600. The T-matrix results for $C_{\text {abs }}[15,16]$ were divided by $\rho V$ and are depicted in blue line. For the case of $\alpha_{a}(a=25 \mathrm{~nm})$, the results computed from GOS/RGD are larger than those from T-matrix by $\sim 6 \%$. 


\section{Concluding remarks}

In this note, the extinction and absorption cross sections, the single-scattering co-albedo, and the asymmetry factor of eight soot fractal aggregates (with primary spheres from 7 to 600) are computed by means of the geometric-optics surface-wave (GOS) approach, coupled with the Rayleigh-Gans-Debye (RGD) adjustment for volume-equivalent size parameters smaller than approximately 2 . The results are compared with those determined from the superposition $\mathrm{T}$ matrix method. Under the condition of random orientation, the single-scattering results determined from GOS/RGD compare reasonably well with those obtained from T-matrix. We further illustrate that using the observed radii of primary spheres ranging from 3.3 to $25 \mathrm{~nm}$, the theoretical values for specific absorption determined from GOS/RGD for primary sphere numbers of 100-600 are within the range of laboratory measured values. Finally, we wish to note that the GOS approach coupled with the RGD adjustment is an attractive and efficient method for the calculation of the single-scattering properties of soot aggregates covering a wide range of size parameters and internal inhomogeneity, particularly for high number of primary spheres (e.g., > 6000) and size parameters much larger than 2.

\section{Acknowledgments}

This research was supported by the National Science Foundation under Grant AGS0946315, by NASA under Grant NNX11AK39, and by Subcontracts S100097 and S100034 from the Texas A\&M Research Foundation. M. Kahnert acknowledges funding support from the Swedish Research Council under Project 621-2011-3346. We are also grateful to D. Mackowski for making his superposition T-matrix code publicly available.

\section{Appendix}

Rayleigh-Gans scattering for a spherical particle is an extension of Rayleigh scattering to particle sizes larger than molecules by taking into account the interference effect from all elements at different positions in a sphere. In order to apply to particles for the real index of 
refraction $m_{r}>>1$, we have replaced the factor $(m-1)^{2}$ in the Rayleigh-Gans scattering intensity [21] by the exact formula for polarizability given by $(9 / 4)\left|\left(m^{2}-1\right) /\left(m^{2}+2\right)\right|^{2}$ such that

$$
I^{R G}=\frac{k^{4} a^{6}}{2 r^{2}}\left|\frac{m^{2}-1}{m^{2}+2}\right|^{2} I_{0} G^{2}(u)\left(1+\cos ^{2} \theta\right),
$$

where $a$ is the radius of a sphere, $k=2 \pi / \lambda, \lambda$ is the wavelength, $r$ is the distance from the scattering particle to an observation point, $m\left(=m_{r}-i m_{i}\right)$ is the complex refractive index, where $m_{r}$ and $m_{i}$ are real and imaginary parts, $i=(-1)^{1 / 2}, I_{0}$ is the incident intensity, and $\theta$ is the scattering angle. According to Gans [22], the factor $G(u)$ is given by

$G(u)=\frac{3}{u^{3}}(\sin u-u \cos u)$,

where $u$ is given by $2 x \sin (\theta / 2)$ and $x$ is the size parameter $k a$. Integrating Eq. (A1) over all directions, we obtain the scattering cross section:

$C_{s c a}^{R G}=\frac{1}{I_{0}} \int I^{R G} r^{2} d \Omega=\pi k^{4} a^{6}\left|\frac{m^{2}-1}{m^{2}+2}\right|^{2} \int_{0}^{\pi} G^{2}(u)\left(1+\cos ^{2} \theta\right) \sin \theta d \theta$.

The asymmetry factor is defined by

$$
g=\frac{\int_{0}^{\pi} I^{R G} \cos \theta \sin \theta d \theta}{\int_{0}^{\pi} I^{R G} \sin \theta d \theta} .
$$

Because the interference effect does not affect absorption, the absorption cross section for Rayleigh-Gans scattering is the same as that for Rayleigh scattering in the form

$$
C_{a b s}^{R G}=-3 k V \operatorname{Im}\left(\frac{m^{2}-1}{m^{2}+2}\right),
$$

where $V$ is the volume.

For an aggregated particle consisting of $N_{s}$ spheres, we may define a form factor $F$ as the ratio of the scattered intensity for $N_{s}$ spheres to that for a single sphere. Debye [23] developed a form factor $F$ in the form

$$
F=\sum_{j=1}^{N_{s}}\left|e^{i \mathbf{q} \cdot \mathbf{r}_{j}}\right|^{2}=\sum_{j=1}^{N_{s}} \sum_{k=1}^{N_{s}} e^{i \mathbf{q} \cdot\left(\mathbf{r}_{j}-\mathbf{r}_{k}\right)}=\sum_{j=1}^{N_{s}} \sum_{k=1}^{N_{s}} \cos \left(\mathbf{q} \cdot \mathbf{r}_{j k}\right)=\sum_{j=1}^{N_{s}} \sum_{k=1}^{N_{s}} \cos \left(|\mathbf{q}|\left|\mathbf{r}_{j k}\right| \cos \eta\right),
$$


where $\mathbf{q}$ is the scattering wave vector and its magnitude is given by $|\mathbf{q}|=2 k \sin (\theta / 2),\left|\mathbf{r}_{j k}\right|=\left|\mathbf{r}_{j}-\mathbf{r}_{k}\right|=r_{j k}$, where $\mathbf{r}_{j}$ and $\mathbf{r}_{k}$ are position vectors of the primary spheres, and $\eta$ is an angle between $\mathbf{q}$ and $\mathbf{r}_{j k}$. Each primary sphere is governed by the Rayleigh-Gans scattering and to a good approximation, it can be considered to be independent of other primary spheres. When an ensemble of aggregates is oriented randomly, we obtain

$I^{R G D}=\int_{0}^{1} I^{R G} F d \cos \eta \cong I^{R G} \int_{0}^{1} F d \cos \eta=I^{R G} \bar{F}$,

where

$\bar{F}=\int_{0}^{1} F d \cos \eta=\sum_{j=1}^{N_{s}} \sum_{k=1}^{N_{s}} \int_{0}^{1} \cos \left(|\mathbf{q}|\left|\mathbf{r}_{j k}\right| \cos \eta\right) d \cos \eta=\sum_{j=1}^{N_{s}} \sum_{k=1}^{N_{s}} \frac{\sin \left[2 k r_{j k} \sin (\theta / 2)\right]}{2 k r_{j k} \sin (\theta / 2)}$.

Accordingly, the scattering cross section is given by

$C_{s c a}^{R G D}=\pi k^{4} a^{6}\left|\frac{m^{2}-1}{m^{2}+2}\right|^{2} \int_{0}^{\pi} G^{2}(u)\left(1+\cos ^{2} \theta\right) \bar{F} \sin \theta d \theta$.

The absorption cross section is simply $N_{s}$ times of the Rayleigh absorption cross section given by $C_{a b s}^{R G D}=N_{s} C_{a b s}^{R G}=-3 N_{s} k V \operatorname{Im}\left(\frac{m^{2}-1}{m^{2}+2}\right)$.

The preceding equations (A7)-(A10) constitute the Rayleigh-Gans-Debye (RGD) approximation for size parameters smaller than about 2 .

\section{References}

[1] Ramanathan V, Carmichael G. Global and regional climate changes due to black carbon. nature geoscience, 2008;1: 221-7, doi:10.1038/ngeo156.

[2] EPA. Report to Congress on Black Carbon 2012. http://www.epa.gov/blackcarbon/ .

[3] Bond TC, Doherty SJ, Fahey DW, et al. Bounding the role of black carbon in the climate system: a scientific assessment. J Geophy Res 2013;118:xxx-yyy, doi: 10.1002/jgrd.50171 (in press).

[4] Liou KN, Takano Y, Yang P. On geometric optics and surface waves for light scattering by spheres. J Quant Spectrosc Radiat Transfer 2010;111:1980-9.

[5] Takano Y, Liou KN. Phase matrix for light scattering by concentrically stratified spheres: comparison of geometric optics and the "exact" theory. Appl Opt 2010;49:3990-6. 
[6] Liou KN, Takano Y, Yang P. Light absorption and scattering by aggregates: Application to black carbon and snow grain. J Quant Spectrosc Radiat Transfer 2011;112:1581-94.

[7] Liou KN, Takano Y, Yue Q, Yang P. On the radiative forcing of contrail cirrus contaminated by black carbon. Geophys Res Lett 2013;40:xxx-yyy, doi:10.1002/grl.50110.

[8] Waterman PC. Matrix formulation of electromagnetic scattering. Proc IEEE 1965;53:805-12.

[9] Mishchenko MI, Travis LD. T-matrix computations of light scattering by large spheroidal particles. Opt Commun 1994;106:16-21.

[10] Laitinen H, Lumme K. T-matrix method for general star-shaped particles: first results. J Quant Spectrosc Radiat Transfer 1998;60:325-34.

[11] Kahnert M. Irreducible representations of finite groups in the T-matrix formulation of the electromagnetic scattering problem. J Opt Soc Am A 2005;22:1187-99.

[12] Mackowski D, Fuller K, Mishchenko M. ftp://ftp.eng.auburn.edu/pub/dmckwski/scatcodes .

[13] Mackowski DW, Mishchenko MI. A multiple sphere T-matrix Fortran code for use on parallel computer clusters. J Quant Spectrosc Radiat Transfer 2011;112:2182-92.

[14] Liu L, Mishchenko MI, Arnott WP. A study of radiative properties of fractal soot aggregates using the superposition T-matrix method. J Quant Spectrosc Radiat Transfer 2008;109:2656-63.

[15] Kahnert M. On the discrepancy between modeled and measured mass absorption cross sections of light absorbing carbon aerosols. Aerosol Sci Technol 2010;44:453-60.

[16] Kahnert M. Numerically exact computations of the optical properties of light absorbing carbon aggregates for wavelength of $200 \mathrm{~nm}-12.2 \mu \mathrm{m}$. Atmos Chem Phys 2010;10:8319-29.

[17] Kahnert M, Nousiainen T, Lindqvist H, Ebert M. Optical properties of light absorbing carbon aggregates mixed with sulfate: assessment of different model geometries for climate forcing calculations. Opt Express 2012;20:10042-58.

[18] Kahnert M, Nousiainen T, Lindqvist H. Models for integrated and differential scattering optical properties of encapsulated light absorbing carbon aggregates. Opt Express 2013;21: 7974-93.

[19] Schmidt K, Yurkin MA, Kahnert M. A case study on the reciprocity in light scattering computations. Opt Express 2012;20:23253-74.

[20] Friedlander SK. Smoke, Dust, and Haze, Fundamentals of Aerosol Dynamics. Second edition. New York: Oxford Univ. Press; 2000.

[21] van de Hulst HC. Light Scattering by Small Particles. New York: Wiley; 1957. 
[22] Gans R. Strahlungsdiagramme ultramikroskopischer Teilchen. Ann Physik 1925;381:29-38.

[23] Debye P. Zerstreuung von Röntgenstrahlen. Ann Physik 1915;351:809-19.

[24] Sheridan PJ, Arnott WP, Ogren JA, et al. The Reno aerosol optics study: an evaluation of aerosol absorption measurement methods. Aerosol Sci Technol 2005;39:1-16.

[25] Bond TC, Bergstrom RW. Light absorption by carbonaceous particles: an investigative review. Aerosol Sci Technol 2006;40:27-67.

[26] Wentzel M, Gorzawski H, Naumann KH, Saathoff H, Weinbruch S. Transmission electron microscopical and aerosol dynamical characterization of soot aerosols. J Aerosol Sci 2003;34:1347-70. 


\section{Figure Captions}

Fig. 1. Black carbon (BC, soot) fractal aggregates containing primary spheres $N_{s}$ of 54,284 , and 600 used for light scattering and absorption calculations.

Fig. 2. Comparison of the extinction and absorption cross sections, the asymmetry factor, and the single-scattering co-albedo for randomly oriented homogeneous soot aggregates between GOS/RGD and T-matrix. GOS and RGD are shown, respectively, by solid and open circles. The total numbers of primary spheres $N_{s}$ depicted in the figure are $7,23,54,105,180,284,432$, and 600 (see Fig. 1). The radius of a primary sphere $a$ is $25 \mathrm{~nm}$. The wavelength is $533.2 \mathrm{~nm}$ with a refractive index of $1.76-i 0.63$. The scale of volume-equivalent size parameter is displayed at the top of the diagrams.

Fig. 3. Comparison of the scattering phase matrix elements for a soot aggregate with $N_{s}=105$ $\left(x_{v}=1.39\right)$ between the Rayleigh-Gans-Debye (RGD) approximation and the superposition Tmatrix.

Fig. 4. Comparison of the specific absorption $\alpha_{a}\left(\mathrm{~m}^{2} / \mathrm{g}\right)$, i.e., the mass absorption cross section (MAC) computed from GOS/RGD for two cases involving the primary-sphere radius of $a=3.3$ and $25 \mathrm{~nm}$ (in red) and the measured values with mean and standard deviation presented by Sheridan et al. [24]. The T-matrix results for $a=25 \mathrm{~nm}$ are also shown (in blue). 
Figure(s)

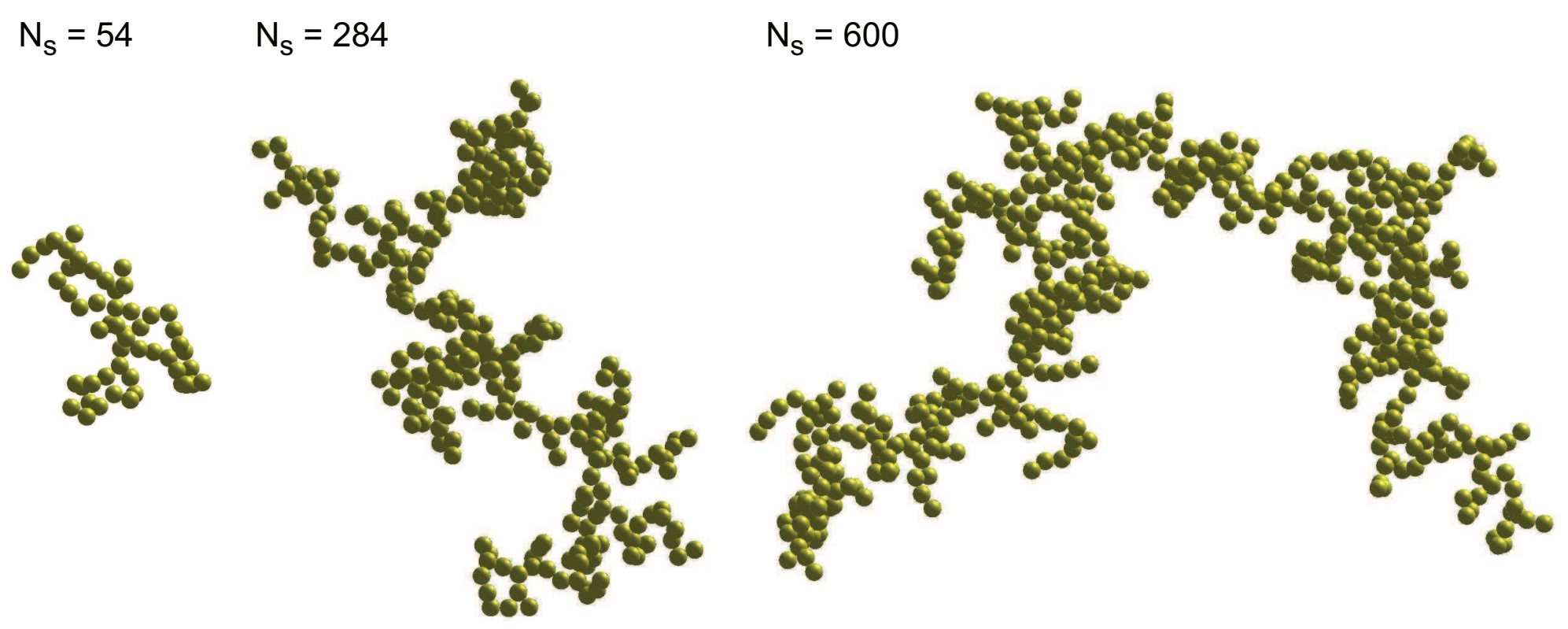

Fig. 1 
Figure(s)

Size Parameter $\mathrm{x}_{\mathrm{v}}$

Size Parameter $\mathrm{x}_{\mathrm{v}}$

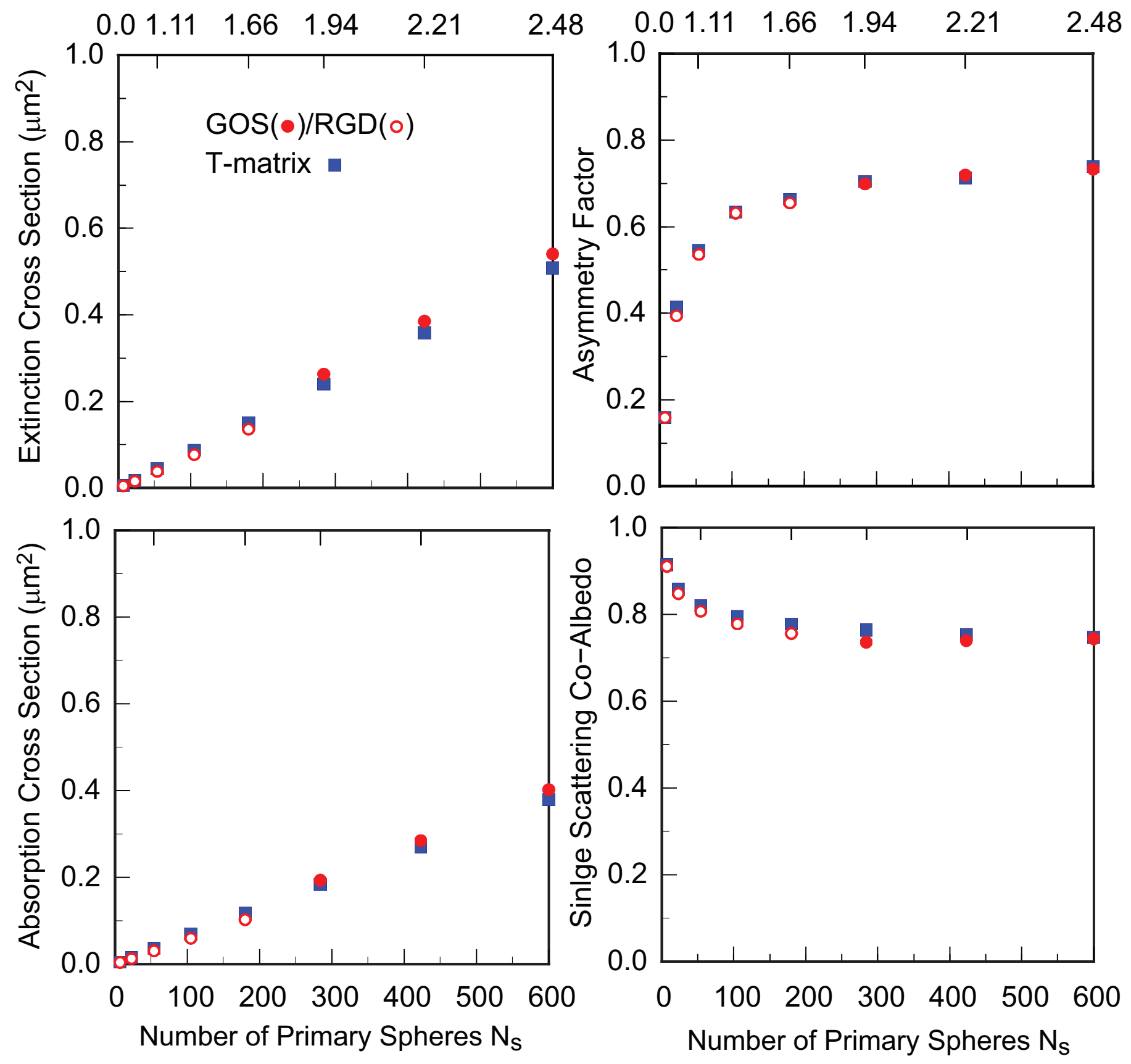

Fig. 2 
Figure(s)

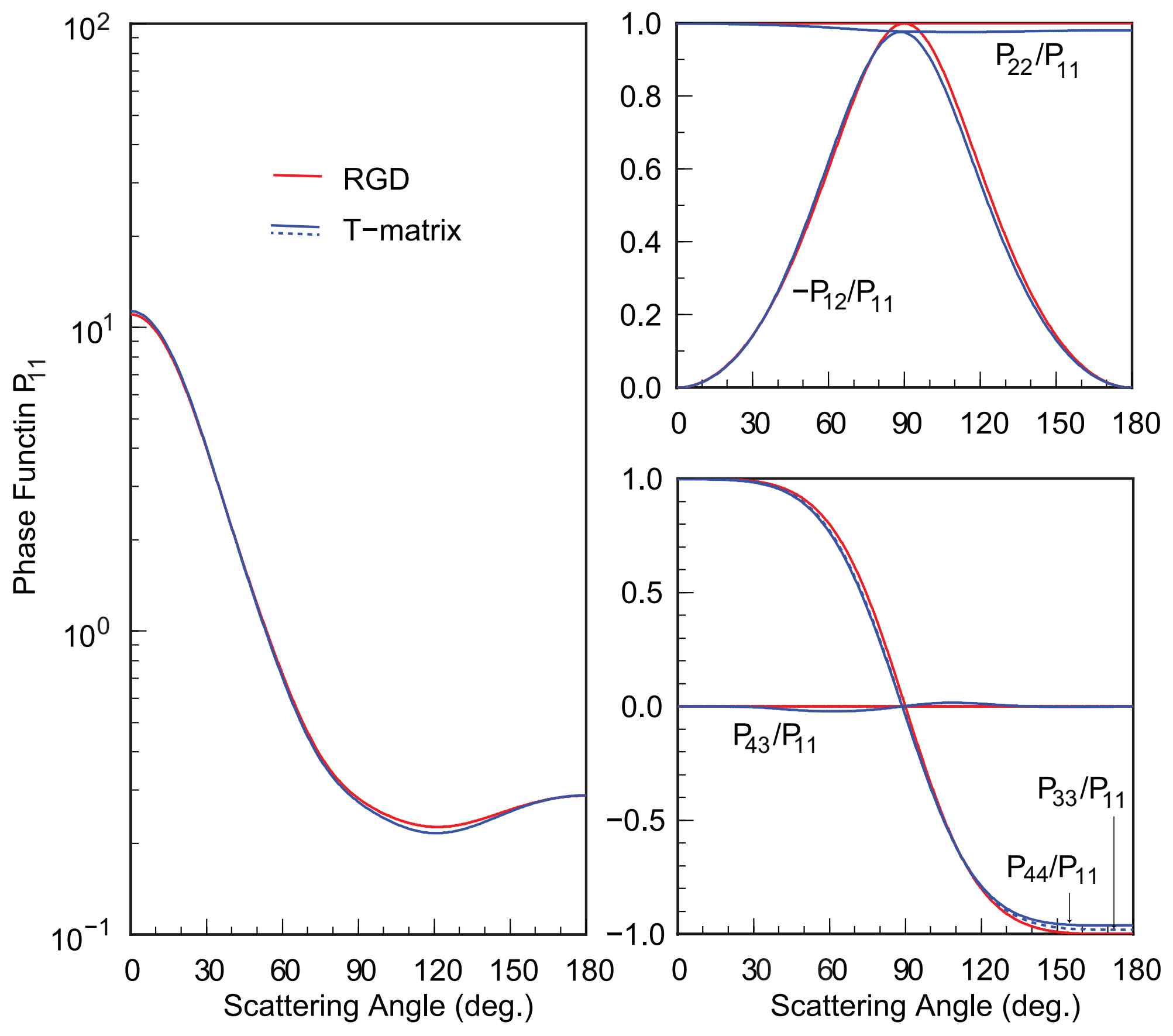

Fig. 3 
Figure(s)

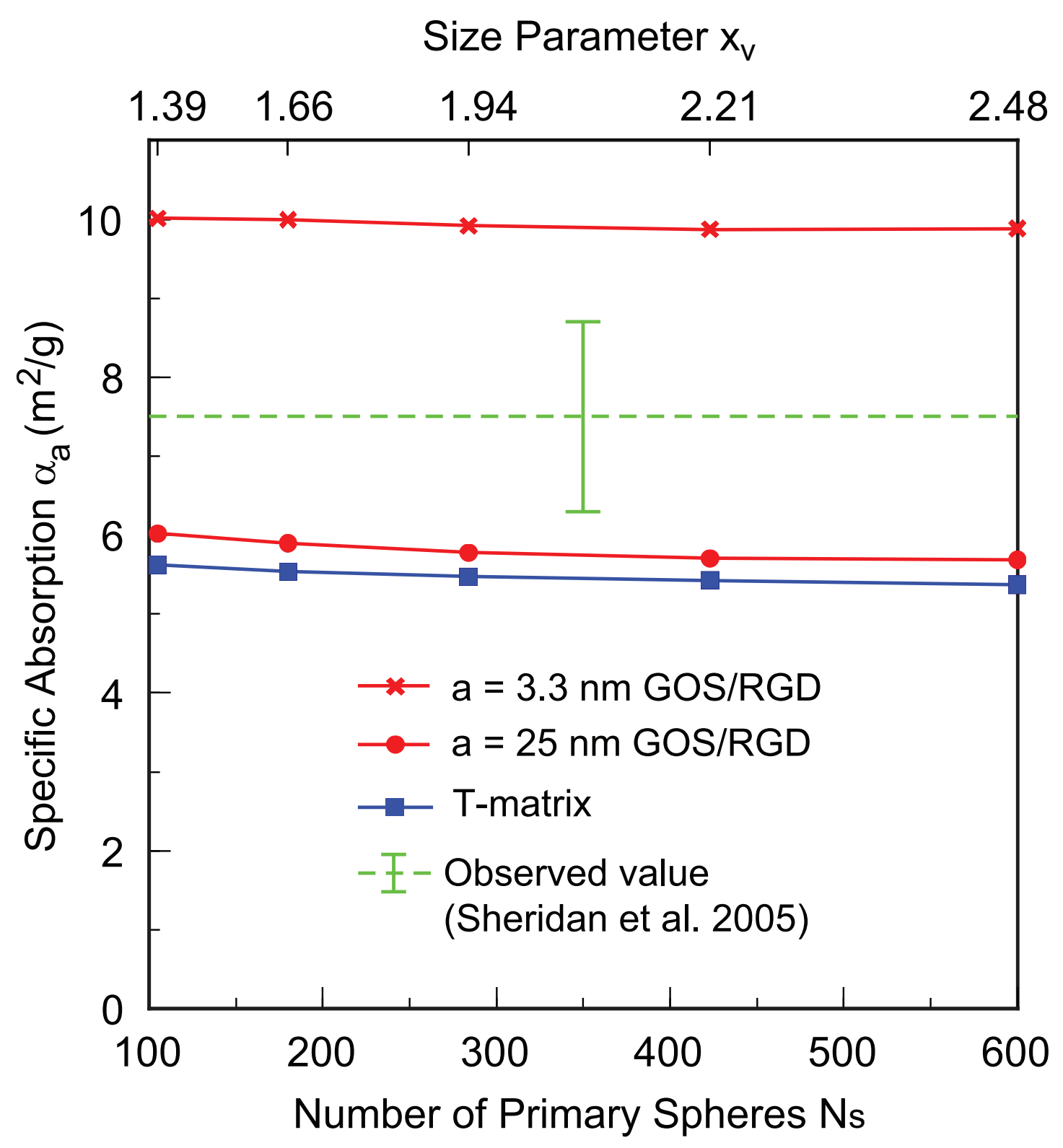

Fig. 4 